\title{
TWINKLE: a low earth orbit visible and infrared exoplanet spectroscopy observatory
}

G. Savini, M. Tessenyi, G. Tinetti, C. Arena, J. Tennyson, et al.

G. Savini, M. Tessenyi, G. Tinetti, C. Arena, J. Tennyson, T. Zingales, En.

Pascale, R. Sudiwala, A. Papageorgiou, S. Sarkar, P. A. R. Ade, M. J. Griffin, K. Barnes, L. Hipwood, P. Knowles, M. Patel, M. Leese, J. P. Mason, M.

Crook, I. Tosh, A. Saad, P. Eccleston, B. Shaughnessy, T. Brooke, M. Wells, I.

Bryson, A. Macleod, W. Taylor, N. Bezawada, G. S. Wright, S. Jason, J.

Friend, J. Williams, G. Johnston, S. Prasad, A. Vora, C. Saunders, B. Winter,

P. Curry, A. Smith, "TWINKLE: a low earth orbit visible and infrared exoplanet spectroscopy observatory," Proc. SPIE 9904, Space Telescopes and Instrumentation 2016: Optical, Infrared, and Millimeter Wave, 99044M (15 March 2018); doi: 10.1117/12.2233691

Event: SPIE Astronomical Telescopes + Instrumentation, 2016, Edinburgh, United Kingdom 


\title{
TWINKLE - A Low Earth Orbit Visible and Infrared Exoplanet Spectroscopy Observatory
}

\author{
The TWINKLE Payload Consortium:
}

$\mathrm{UCL}^{\mathrm{a}}$ - G. Savini, M. Tessenyi, G. Tinetti, C. Arena, J. Tennyson, T. Zingales

Cardiff University $^{\mathrm{b}}$ - E. Pascale, R. Sudiwala, A. Papageorgiou, S. Sarkar, P.A.R. Ade, M. J. Griffin

LEONARDO $^{c}-$ K. Barnes, L. Hipwood, P. Knowles

Open University ${ }^{\mathrm{d}}$ - M. Patel, M. Leese, J.P. Mason

STFC RAL Space ${ }^{\mathrm{e}}$ - M. Crook, Technology Department, I. Tosh, A. Saad, P. Eccleston, B. Shaughnessy, T. Brooke

STFC UKATC ${ }^{\mathrm{f}}$ - M. Wells, I. Bryson, A. MacLeod, W. Taylor, N. Bezawada, G. S. Wright

SSTL (Surrey Satellite Technology Ltd.) ${ }^{g}-$ S. Jason, J. Friend, J. Williams, G. Johnston, S. Prasad, A. Vora, C. Saunders

$\mathrm{UCL} \mathrm{MSSL}^{\mathrm{h}}-$ B. Winter, P. Curry, A. Smith

${ }^{a}$ Physics \& Astronomy Dept., University College London, Gower Street, WC1E 6BT, London, UK;

${ }^{\mathrm{b}}$ School of Physics \& Astronomy, Cardiff University, Cardiff CF24 3AA, Cardiff, Wales UK;

'LEONARDO, UK;

${ }^{\mathrm{d}}$ Department of Physical Sciences, Open University, Milton Keynes UK;

${ }^{\mathrm{e}} \mathrm{STFC}$ - Rutherford Appleton Laboratory, Harwell, UK;

${ }^{\mathrm{f}}$ STFC - UK Advanced Technology Centre, Edinburgh, Scotland, UK;

${ }^{\text {g} S u r r e y ~ S a t e l l i t e ~ T e c h n o l o g y ~ L t d ., ~ G u i l d f o r d, ~ S u r r e y, ~ E n g l a n d, ~ U K ; ~}$

${ }^{\mathrm{h}}$ Mullard Space Science Laboratory, Surrey, England, UK

This is a preprint of a SPIE Proceedings paper (Astronomical Telescopes + Instrumentation conference, 2016, [9904175]), and is linked to two other SPIE publications relevant to Twinkle: M.Wells [9904-176] and S.Sarkar [9904-138]

\begin{abstract}
Twinkle is a space mission designed for visible and near-IR spectroscopic observations of extrasolar planets. Twinkle's highly stable instrument will allow the photometric and spectroscopic observation of a wide range of planetary classes around different types of stars, with a focus on bright sources close to the ecliptic. The planets will be observed through transit and eclipse photometry and spectroscopy, as well as phase curves, eclipse mapping and multiple narrow-band time-series. The targets observed by Twinkle will be composed of known exoplanets mainly discovered by existing and upcoming ground surveys in our galaxy (e.g. WASP, HATNet, NGTS and radial velocity surveys) and will also feature new discoveries by space observatories (K2, GAIA, Cheops, TESS).

Twinkle is a small satellite with a payload designed to perform high-quality astrophysical observations while adapting to the design of an existing Low Earth Orbit commercial satellite platform. The SSTL-300 bus, to be launched into a lowEarth sun-synchronous polar orbit by 2019 , will carry a half-meter class telescope with two instruments (visible and near-IR spectrographs - between 0.4 and $4.5 \mu \mathrm{m}$ - with resolving power $\mathrm{R} \sim 300$ at the lower end of the wavelength scale) using mostly flight proven spacecraft systems designed by Surrey Satellite Technology Ltd and a combination of high TRL instrumentation and a few lower TRL elements built by a consortium of UK institutes.
\end{abstract}

Space Telescopes and Instrumentation 2016: Optical, Infrared, and Millimeter Wave, edited by Howard A. MacEwen, Giovanni G. Fazio, Makenzie Lystrup, Proc. of SPIE Vol. 9904, 99044M

(c) The Authors. Published under a Creative Commons Attribution CC BY 3.0 License · doi: 10.1117/12.2233691 
The Twinkle design will enable the observation of the chemical composition and weather of at least 100 exoplanets in the Milky Way, including super-Earths (rocky planets 1-10 times the mass of Earth), Neptunes, sub-Neptunes and gas giants like Jupiter. It will also allow the follow-up photometric observations of 1000+ exoplanets in the visible and infrared, as well as observations of Solar system objects, bright stars and disks.

Keywords: Exoplanets, Space telescopes-IR, Innovative space telescopes and technologies.

\section{INTRODUCTION}

The Twinkle satellite project is a commercially based mission with the aim of addressing the high demand from academic and research institutes across the world, from government bodies aiming to raise the profile of the STEM subjects in their national education program by providing high quality scientific observations via an affordable mission assembled in a relatively short timescale designed to achieve nominal scientific requirements while at the same time being compliant with a "standard" satellite platform with high heritage [1]. The idea behind the payload design is to be guided by the science requirements with a platform that has the potential of integration and assembly within the timeframe when Twinkle can have the highest impact ( $\sim 4$ years). As a result, the Payload Design exercise performed a number of iterations between the science requirements and the readiness and availability of the hardware, which will deliver the highest grade of science.

\subsection{Science Payload Requirements}

The payload is constituted by a telescope and science instruments covering visible and near infrared wavelength ranges. It will address pointing stability by including an additional Fine-Guidancesystem in the payload design as well as the necessary thermal architecture to ensure that the NIR instrument detectors operate with sufficient sensitivity. An overall layout and interface to a modified design of the SSTL-300 platform is also discussed.

The science requirements for the payload instruments are set by the inclusion of a significant number of spectroscopic lines of molecules of interest [2,3] and corresponding spectral resolution that will allow to decouple the presence of different molecular species. The resulting characteristics of the payload and its instruments can be found in Table 1. The scientific requirements are based on previous work [4]. A detailed science case for Twinkle will be published in a subsequent paper.

Table 1. Basic payload characteristics derived from science requirements and compliant with the mass and volume of an SSTL-300 platform.

\begin{tabular}{|l|l|l|l|}
\hline Parameter & Value & Unit & Comment \\
\hline $\begin{array}{l}\text { Visible channel } \\
\text { Wavelength range }\end{array}$ & $0.4-1.0$ & $\mu \mathrm{m}$ & \\
\hline $\begin{array}{l}\text { IR channel } \\
\text { Wavelength range }\end{array}$ & $1.3-4.5$ & $\mu \mathrm{m}$ & $\begin{array}{l}\text { Split into } 2 \text { bands } \\
1.3-2.42 \mu \mathrm{m} \text { and } 2.42-4.5 \mu \mathrm{m}\end{array}$ \\
\hline Resolving power & 300 & 70 & $\begin{array}{l}<2.4 \mu \mathrm{m} \\
>2.4 \mu \mathrm{m}\end{array}$ \\
\hline $\begin{array}{l}\text { Detector background } \\
\text { area }\end{array}$ & $10 \mathrm{x}$ & In two adjacent strips of sky \\
\hline $\begin{array}{l}\text { Primary mirror } \\
\text { diameter }\end{array}$ & 450 & $\mathrm{~mm}$ & \\
\hline
\end{tabular}




\begin{tabular}{|l|l|l|l|}
\hline $\begin{array}{l}\text { Field-of-View of } \\
\text { FGS }\end{array}$ & \pm 3 & arcmin & $\begin{array}{l}\text { Allows the tip-tilt mirror to correct for } \\
\text { pointing jitter. }\end{array}$ \\
\hline $\begin{array}{l}\text { Overall optical } \\
\text { efficiency }\end{array}$ & 0.4 & & $\begin{array}{l}<2.4 \mu \mathrm{m} \\
>2.4 \mu \mathrm{m}\end{array}$ \\
\hline Total payload mass & $<100$ & $\mathrm{Kg}$ & $\begin{array}{l}\text { Including cryo-coolers, baffles and payload } \\
\text { electronics units }\end{array}$ \\
\hline $\begin{array}{l}\text { Average payload } \\
\text { power }\end{array}$ & $<100$ & $\mathrm{~W}$ & $\begin{array}{l}\text { Including cryo-coolers and payload } \\
\text { electronics units }\end{array}$ \\
\hline
\end{tabular}

\subsection{Payload performance estimation method}

The performance of the instruments which are part of the payload is assessed using a radiometric model (Cardiff Radiometric Model, CRM) and ExoSim [4,5]. ExoSim is an end-to-end time-domain instrument simulator, which uses a parametric description of both the instrument and the astronomical scene to simulate the spectroscopic detection of transiting planets' light-curves. ExoSim captures astrophysical and instrumental noise sources and systematics providing the most realistic description of TWINKLE's science capabilities. Figure 1-1 shows a simulated spectrum of a hot Jupiter as seen by Twinkle, obtained with ExoSim.

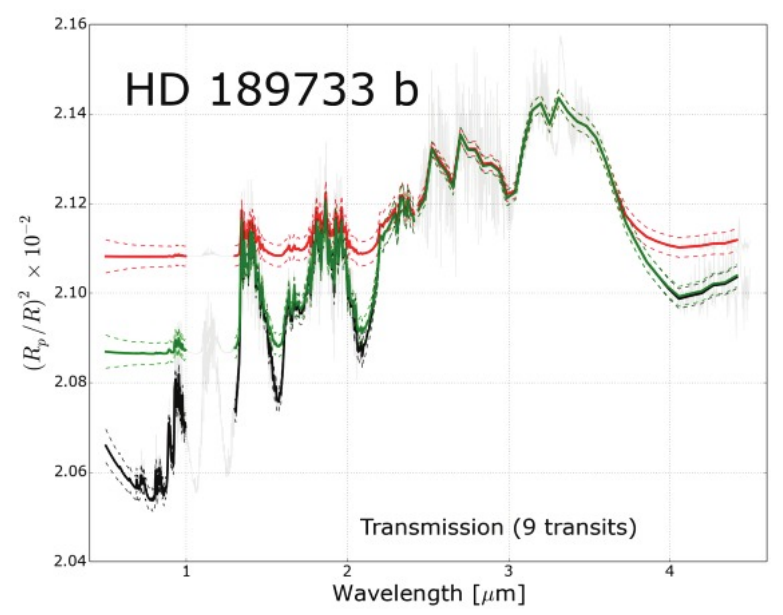

Figure 1-1: Simulated spectrum of transiting hot Jupiter HD189733b with models showing cloud signatures in the $0.5-3 \mu \mathrm{m}$ range, as observed by TWINKLE. The simulation was obtained with our end-to-end simulator ExoSim. Through these measurements TWINKLE will detect molecules, the presence of clouds/hazes, and constrain cloud parameters such as altitude, thickness, particle sizes in the atmospheres of over 100 exoplanets.

The CRM provides a static (non-time-domain) simulation of the detection by implementing a radiometric description of the coupling of the instrument with the focal plane detector array as well as a representative description of the most important noise sources and methods utilised to reconstruct the spectral information from measurements. The CRM is most useful for studying the relative importance of noise sources in the instrument noise budget and performing quick estimates of the confidence of the detection. Temperature limits on critical subsystems for example have been set this way. For a more detailed description of ExoSim see [5]. 


\section{TELESCOPE DESIGN}

\subsection{Design rationale}

There are a number of significant design drivers for the TWINKLE telescope:

- A minimum entrance pupil diameter of $450 \mathrm{~mm}$ with a low obscuration ratio to meet the flux requirements.

- An accessible exit pupil where a steering mirror can be located for tracking during observations (due to the limitations of the spacecraft stability).

- Sufficient space around the focal plane position to accommodate the IR spectrometer, visible spectrometer, FGS and dichroics.

- An F\#>10 input to match the spectrometer requirements

- An exit pupil to image plane distance of $\sim 250 \mathrm{~mm}$ to match the spectrometer input requirements.

- $\mathrm{FOV} \pm 3^{\prime}$ (up to $5^{\prime}$ ) with minimal variation of the image quality.

- Overall length to keep within the SSTL platform constraints and low cost launch option compatibility.

- Wavelength range $0.5 \mu \mathrm{m}<\lambda<4.5 \mu \mathrm{m}$

- Good straylight control

Of the available designs considered, those without an accessible exit pupil and any with refractive components can be discounted e.g. the Topsat TMA and Cassegrain/corrector types. It became apparent during the study that the best available solution was a Korsch type TMA. These designs are being used extensively in Earth Observation camera systems, including a Rutherford Appleton Laboratory Space camera - RALCAM4 [6]. Fortuitously, RALCAM4's specification already closely matches those above for TWINKLE so was an obvious choice to use as a basis to develop the TWINKLE design.

The optical layout and the CFRP structural model of RALCAM4 are shown in Figure 2-1 below:

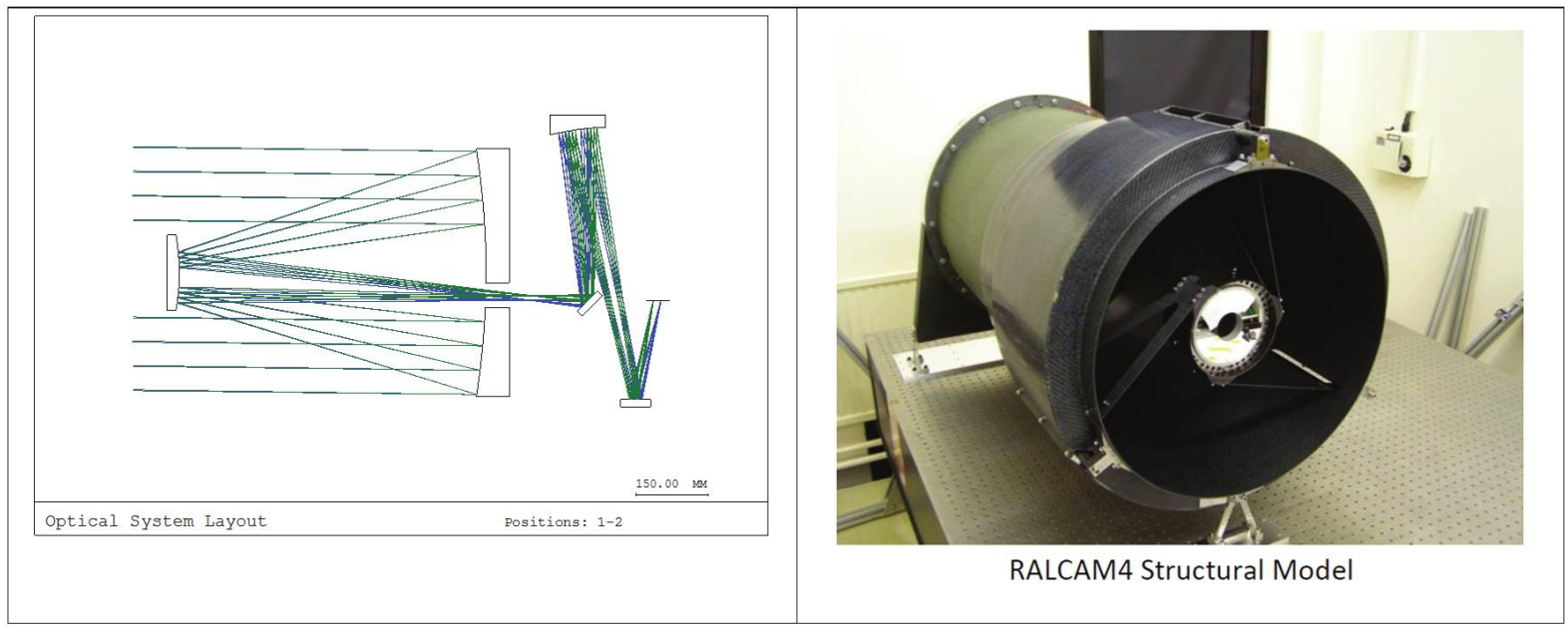

Figure 2-1: (Left) RALCAM 4 optical TMA design. (Right) The RALCAM 4 STM. 


\subsection{Design Description}

Although the RALCAM4 design could easily deliver an image quality close to the diffraction limit (in the visible) it required significant tweaking to ensure that the pupil position and packaging requirements could be met. The final design is shown in Figure 2-2.

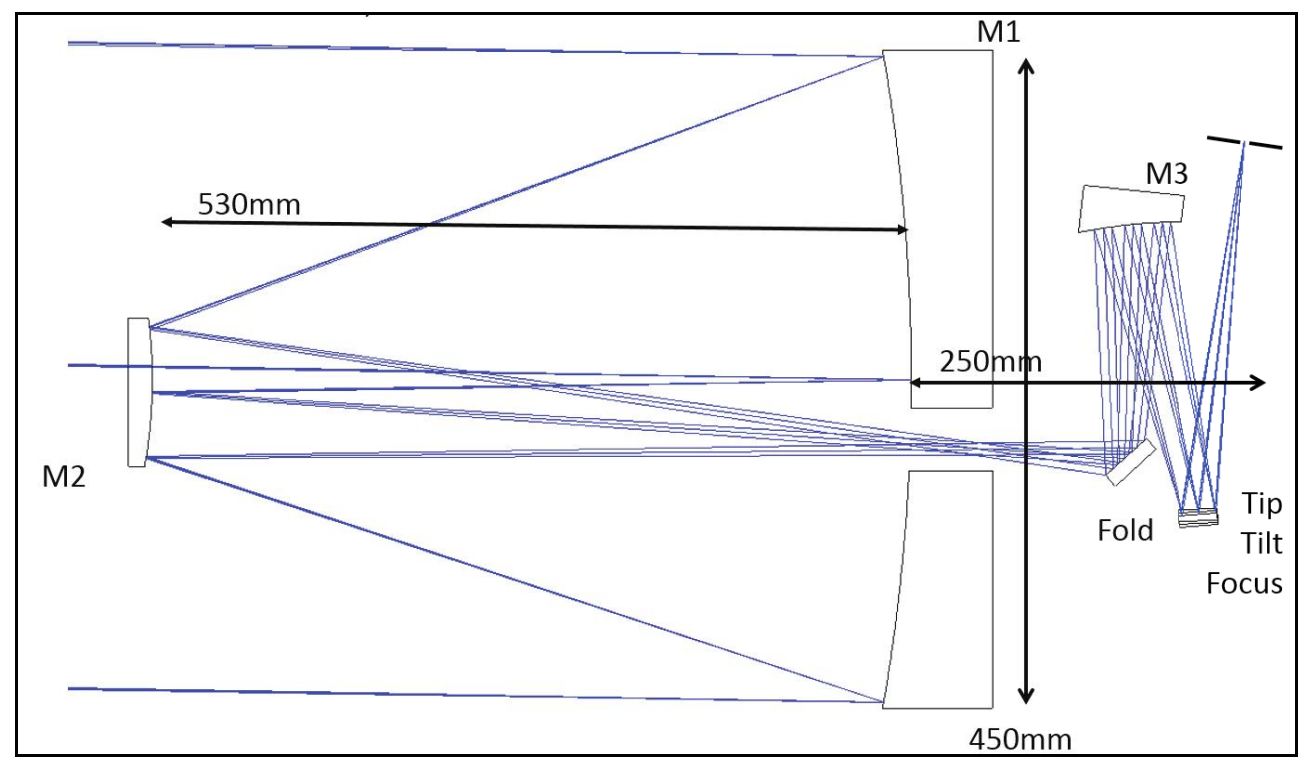

Figure 2-2. Current optimized TWINKLE optical design. The Focal Plane position is brought further up, the fold anticipated for envelope reasons and an image of the pupil established at the tip-tilt mirror.

The telescope design is based on an annular field Korsch telescope which has an obscured central field. In this case we use an off-set field of $\sim 1^{\circ}$ to use an unobscured FOV and to ensure that there aren't any beam clashes with the other mirrors. For packaging, there is an added fold mirror just behind the primary mirror (M1) which is close to the intermediate image plane. The final flat mirror at the exit pupil provides tip/tilt/focus control. and also allow the system to be folded within the space envelope required. The tip/tilt mirror can be controlled to keep the star image static (using the FGS) at the input to the spectrometers.

\subsection{Secondary mirror mount and alignment}

There are two current options for the mount of the secondary mirror. The first mounting solution adopts the RALCAM4 design based on three CFRP struts which link the secondary to one of the bulkheads surrounding the telescope baffle. This solution is shown in figure in the RALCAM4 telescope design alone and within the SSTL-300 platform. In the same figure, the mounting structure of the RALCAM4 can be seen on the "bottom side" of the baffle structure. This is not the foreseen telescope mount interface to the platform (see mechanical interface section).

The second solution adopts metering rods which would allow good thermal expansion at the expense of mount complexity given the reduced size of the secondary mirror. 

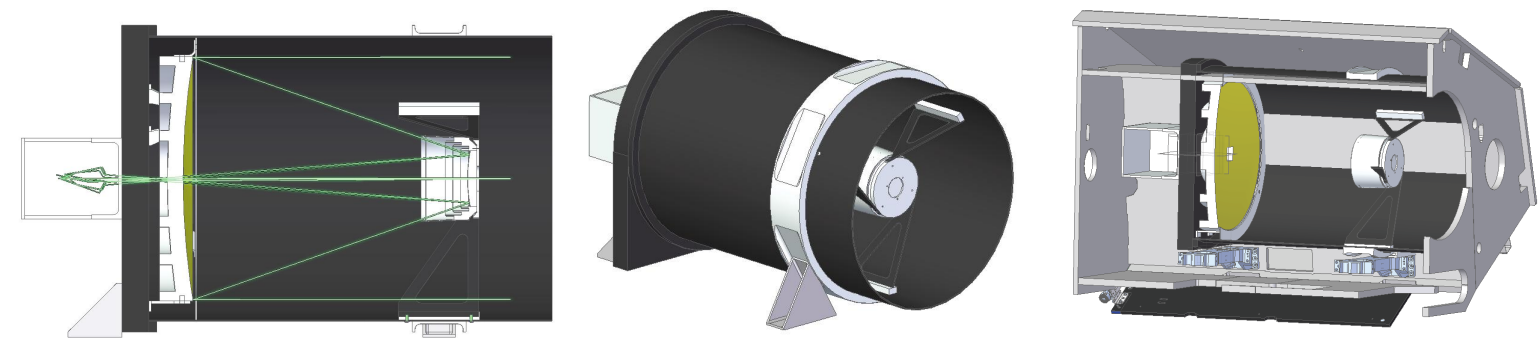

Figure 2-3. Left and Centre: The preliminary RALCAM4 mounting option with a bulkhead close to the front of the telescope baffle, allowing stiffer support of the secondary and the mounting struts. Right: Housed in the SSTL-300 platform. This is just a concept drawing. The mounting of the baffle to the platform is suggested later to occur via two connected bulkheads and along the sides of the platform.

\section{EXOPLANET LIGHT VISIBLE INSTURMENT (ELVIS)}

\subsection{Philosophy and heritage from UVIS/NOMAD.}

The Exoplanet Light VIsible Spectrometer (ELVIS) is a visible spectrometer channel based on the Ultraviolet and Visible Spectrometer (UVIS) instrument channel launched on the ExoMars Trace Gas Orbiter (TGO) in March 2016. For TGO, UVIS measures ozone, dust and aerosols in the martian atmosphere via observations of the Mars surface (in nadir mode) or the sun (in occultation mode) through the atmosphere.

The spectrometer is based on a Czerny-Turner optical configuration. The spectrometer configuration combines simplicity and high-performance, with a broad spectral range of $200-650 \mathrm{~nm}$ and optimized performance in the UV range. Internally the spectrometer uses a UV coated back-illuminated e2V CCD 30-11 as its detector and a grating that gives UVIS a spectral range of 200-650 nm at a resolution of $\sim 1.5 \mathrm{~nm}$. The combined instrument has a signal to noise ratio (SNR) of $>500$ in Nadir and $>1000$ in SO. An image of the UVIS flight model is shown in Figure 3-1.

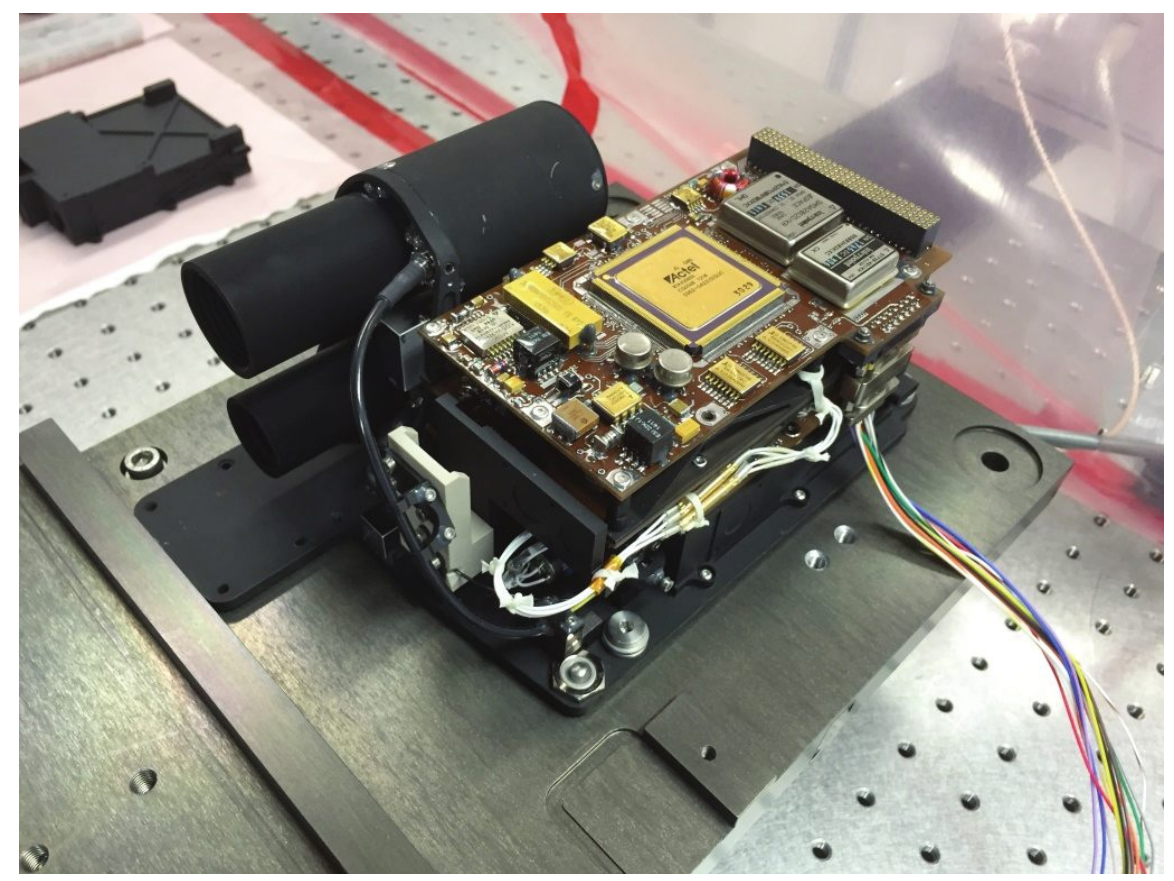

Figure 3-1 UVIS Flight Model instrument under test 


\subsection{Optical spectrometer (modifications to UVIS)}

For the Mars application the UVIS instrument used a dual telescope configuration; nadir (downward viewing of the surface for total atmospheric column measurements) and solar occultation observations (SO, looking at the Sun through the atmosphere from orbit to measure vertical profiles). The telescopes were connected to a single spectrometer via a fibre optic selector link. This telescope and selector system is not required in the Twinkle application as the spectrometer is positioned in the visible beam of the main Twinkle telescope.

The main modification to the spectrometer design is the use of an alternative grating and associated coatings to optimize the spectral range to the visible to near IR range between 0.4 and 1.0 micron.

Other planned changes include a minor electronics component change on the detector board and relocation of the main electronics board stack to improve thermal isolation and allow the detector to run at a lower temperature.

Changes to the firmware code within the electronics will optimize the operations (e.g. CCD readout modes) and integration times for the Twinkle application.

\subsection{Detectors and readout}

The CCD30-11 is an advanced inverted mode operation (AIMO) back-Illuminated (BI) high performance three-phase buried channel CCD; details on the CCD are provided in Table 2. The CCD was produced using the $2 \mathrm{v}$ quantum efficiency $(\mathrm{QE})$ enhanced process with an optical coating applied to the CCD to increase the QE over the wavelength range. The addition of a boron implant under the integrating electrode defines a fixed potential well when the electrodes are all held at $0 \mathrm{~V}$, which allows charge collection to be performed with the whole surface inverted minimizing dark current generation and allowing the device to be operated at warmer temperatures.

\subsection{Performance and detector parameters}

Table 2. Basic characteristics of the modified visible camera for Twinkle from the UVIS instrument.

\begin{tabular}{|c|c|}
\hline Wavelength range & $400-1000 \mathrm{~nm}$ \\
\hline Resolution & $\sim 2 \mathrm{~nm}$ \\
\hline Detector: Image area & $26.6 \times 6.7 \mathrm{~mm}$ \\
\hline Active pixels (H) & 1024 \\
\hline Active pixels (V) & 255 \\
\hline Under-scan & 8 pixels \\
\hline Over-scan & $26 \times 26 \mu \mathrm{m}$ \\
\hline Pixel size & $26 \mathrm{~s}$ \\
\hline
\end{tabular}

\section{INFRARED INSTRUMENT}

The Infra-Red Science Instrument is on the other hand an original design, the details of which are outlined in full in a sister paper in this conference [7]. The goal of this design was to allow from a single incoming high F-number beam, the split in two separate channels (given the broadband nature 
of the desired spectral coverage) each with an appreciable spectral resolution (initially of the order of $\sim 100$ or more).

The use of a narrow slit for the source and background to reduce the amount of stray-light by facilitating baffling internally within the instrument was suggested initially and also evolved in the iterations to a separate slit for the source and for the background field for subtraction.

Finally, given that the final portion of the identified detector to be used was relatively contained to less than half of a full array, and that discussions with the detector manufacturers identified the possibility of producing separate coatings for two portions of the detector, the design was further compacted and modified so that the two channels would make use of different halves of the same detector therefore reducing costs substantially.

\subsection{Instrument Optical Design}

The IR Instrument consists of two spectrometers which are intricately connected even though they only share one common optical element within the instrument (after the telescope optical train). The spectrometers work by delivering diffraction limited image over two spectral bands, 1.3-2.42 $\mu \mathrm{m}$ and 2.42-4.5 $\mu \mathrm{m}$ with resolving powers of $\mathrm{R}=300$ and $\mathrm{R}=70$ respectively. Figure $4-1$ shows a perspective ray-tracing of the instrument albeit a full set of projections is needed to fully appreciate the propagation of rays through the optical system starting from the prism beam-splitter and ending on a common detector.

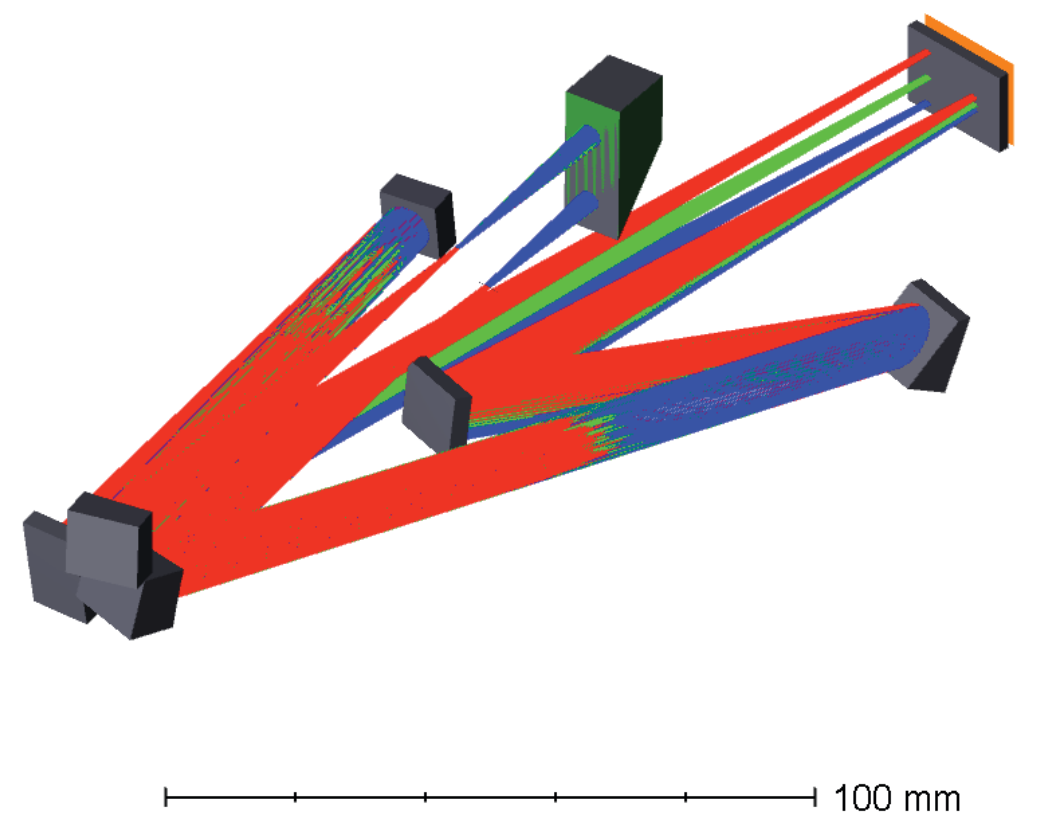

Figure 4-1. Perspective view of the IR instrument with the "entrance" prism seen in the top centre of the picture. The rays enter vertically from the top and the two outputs (reflected and transmitted in the prism) exit the prism towards the bottom left of the viewer. The multitude of rays seen encompass the three wavelengths per channel and the centres of the 3 fields (star + background fields on both sides of the star.

The layout of the optics of the two channels of the instrument has been checked for non-invasive presence of optical elements in each other's beams.

The nature of the input rays to the detector is such that no small-aperture enclosure can separate the detector arrays from the rest of the instrument. But given that a lengthy side baffle reduces the 
throughput incoming from the warmer Instrument box shield by a considerable amount, a rectangular section shield is added for this purpose

\subsection{Inner optics}

The spectrum of the star is separated into Channel $1(1.3$ to $2.42 \mu \mathrm{m})$ and Channel 2 (2.42 to $4.5 \mu \mathrm{m})$ by the CaF2 dichroic prism. There are instrument slit masks in each channel that act to limit the background flux entering the spectrometer. For light from the star and associated planet(s) the PSF is the effective slit width while for the background the physical width of the slit is used. These slit widths combined with dispersing element, collimator and camera mirrors and detector pixels determine the spectral resolving power.

The length of the slits is determined by the available area of the detector, this is 60 arcsec for Channel 1 and 112 arcsec for Channel 2. The slit mask also represents the only access to an enclosed area which effectively shields most of the instrument from stray light reducing the constraints on the telescope baffle.

Table 3 The Airy PSF angular and linear sizes at the instrument entrance slit.

\begin{tabular}{|l|l|l|l|l|}
\hline Wavelength & $\begin{array}{l}2.44 \\
\lambda / \mathrm{D}\end{array}$ & $\begin{array}{l}\text { Linear } \\
\text { size at slit }\end{array}$ & $\lambda / \mathrm{D}$ & $\begin{array}{l}\text { Linear } \\
\text { size at slit }\end{array}$ \\
\hline$\mu \mathrm{m}$ & $\operatorname{arcsec}$ & $\mathrm{mm}$ & $\operatorname{arcsec}$ & $\mathrm{mm}$ \\
\hline 1.3 & 1.45 & 0.036 & 0.60 & 0.015 \\
\hline 2.4 & 2.68 & 0.067 & 1.10 & 0.027 \\
\hline 4.5 & 5.03 & 0.126 & 2.06 & 0.051 \\
\hline
\end{tabular}

The beams leaving the 2 entrance slits are collimated by 2 diamond turned mirrors which in turn direct light towards the dispersing elements, a diffraction grating for Channel 1 and a calcium fluoride prism for Channel 2. The spectra are then imaged on to the detector by 2 more diamond turned mirrors. The spectra are aligned to lie along columns of pixels by rotating the grating/prism about their optical axes.

Detector arrays such as those proposed for Twinkle can present a variation in the QE (gain) at the sub-pixel level. This means that jitter in the star position along the entrance slit can result in appreciable changes in signal level. To reduce this effect an array of cylindrical lenses is placed in front of the detector that, in the cross-dispersion direction, image the telescope pupil onto the detector. This means that the movement of the star at the instrument entrance focal plane is translated into a change of angle of incidence at the detector, which results in a smaller change in signal than a direct lateral shift.

\subsection{Infrared Leonardo Detectors}

The two options considered for the IR channel detector are:

- separately optimised 2.5 and $5 \mu$ m cut-off FPAs on the same type of ROIC for channels 1 and 2 respectively (option 1 );

- a single FPA with two optimised MCT sub-arrays (option 2). 
Both options provide the possibility of electronically windowing the full format array down to one or more rectangular regions of interest. A third option, a single full array with a $1.3-5 \mu \mathrm{m}$ spectral response, could also be considered in principle. This structure has not been grown or assessed previously, so a trial would be needed before commitment to this approach. In this case channel 1 would also have higher dark current than options 1 and 2, and the achievement of an antireflection coating covering the $1.3-5 \mu \mathrm{m}$ interval may be problematic. The 2-stage coating of separate MCT windows, as in option 2 , remains a valid approach.

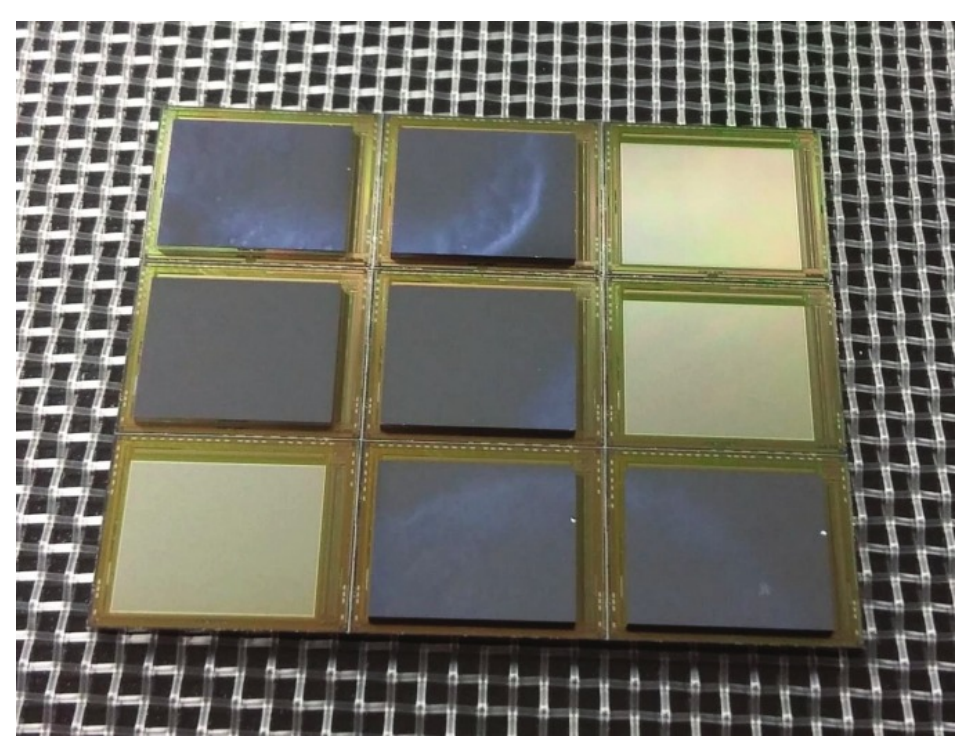

Figure 4-2. Multiple arrays on a block of ROICs.

\section{Option 1}

Separate full-size arrays represent the simplest manufacturing option. This is a duplication of previous work for the $2.5 \mu \mathrm{m}$ cut-off array, assuming a single layer $1 / 4$-wave antireflection coating is applied. The $5 \mu \mathrm{m}$ cut-off array is identical in design to the short wave array apart from the crystal growth structure, which is a common growth recipe for a wide range of tactical arrays, and the thickness of the antireflection coating. Separate arrays provide independent diode bias voltages and stare time control for the two channels. In principle this solution allows the two arrays to be operated at different temperatures.

\section{Option 2}

A single FPA with two MCT sub-arrays provides a more compact detector with half the power dissipation of option 1.

Typical spectral responses at $77 \mathrm{~K}$ are shown in Figure 4-3 for an array that approximates the TWINKLE requirements. 


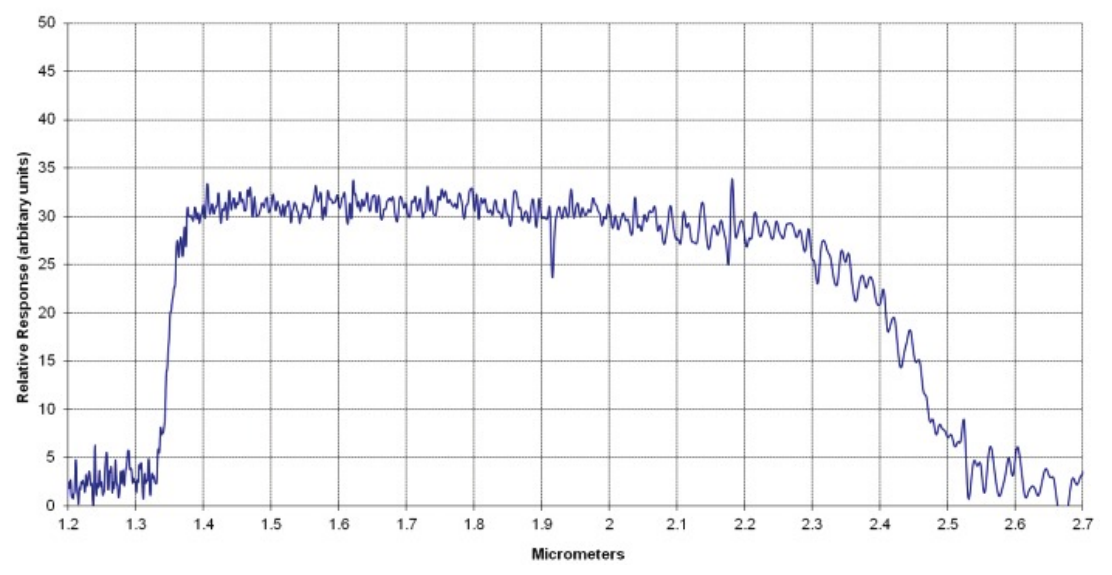

Figure 4-3. Spectral response of 1.35-2.45 $\mu \mathrm{m}$ array (at $50 \%$ cut-on and cut-off).

Fine tuning of dedicated wafer growths will allow the spectral coverage to be optimised to achieve high quantum efficiency across each of the two bands. The wavelength targets for channel 2 need to take into account the planned operating temperature. Figure 4-4 below shows the variation of MCT cut-off with temperature. The temperature dependence of channel 1 is much weaker.

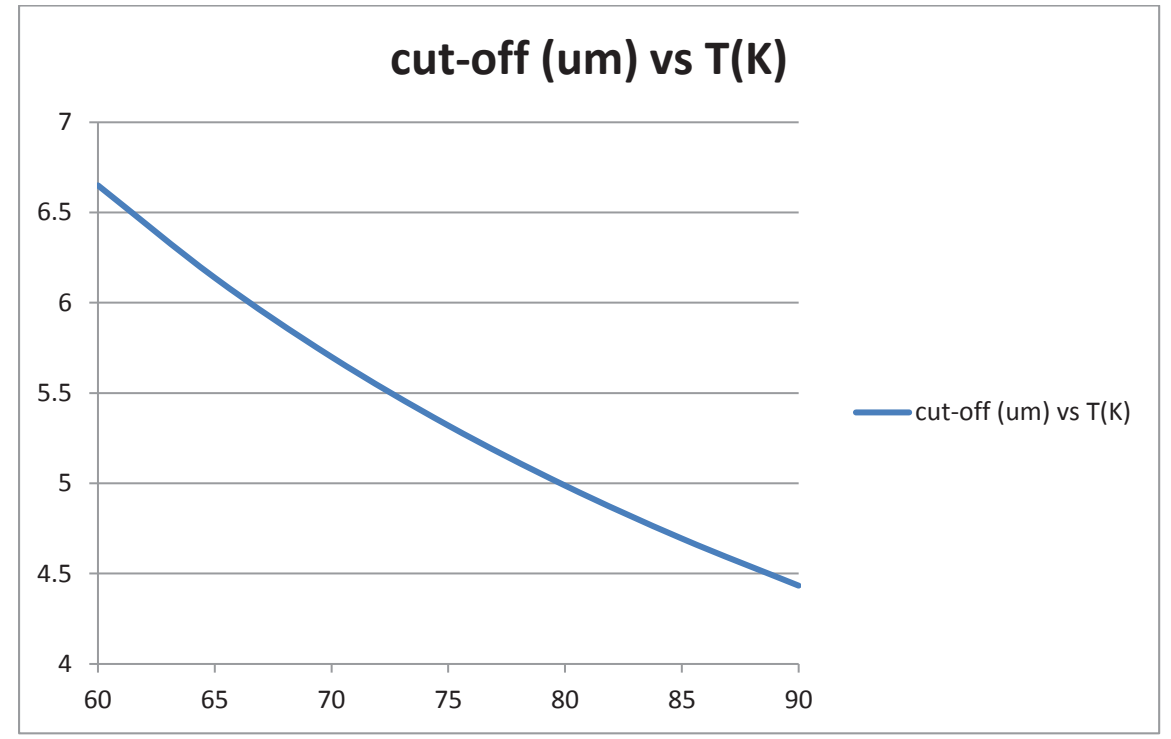

Figure 4-4. Cut-off vs temperature for MCT composition with 5 microns at $80 \mathrm{~K}$.

In the plateau regions of the signal response, QE is estimated to be at least $70 \%$ (including fill factor) with a $1 / 4$-wave antireflection coating. Higher QE could be achieved through multilayer antireflection coating (MLARC) and, in the case of channel 2 only, by growing a thicker 'absorber' layer within the diode heterostructure, but at the expense of increased dark current in proportion to the increased absorber thickness.

\subsection{ROIC}

The planned Read-Out Integrated Circuit for the above detector is the Leonardo ME930 ROIC. The pixel circuit concept is based on a floating gate input cell to meet the low noise and low power dissipation requirements, as implemented on the ME930 and ME950 ROICs developed for ESA. 
The key parameters and features of the ME930 include:

- $0.35 \mu \mathrm{m}$ CMOS technology

- $15 \mu \mathrm{m}$ pitch

- Floating gate input cell

- 1276 rows x 1024 columns active pixel array

- Rolling Shutter non-destructive readout mode

- reference rows (4 top and 4 bottom)

- reference columns (2 left and 2 right)

- Programmable outputs: $4,8,16$ or 32

- Programmable readout region

- SPI electrical interface

- Radiation hard cell library

The designs have implemented a radiation hard cell library. Heavy ion radiation testing has been successfully completed on the ME930 ROIC under UKSA funding. The ROIC is immune to latch-up to LET values of at least $67 \mathrm{MeV} / \mathrm{g} / \mathrm{cm}^{2}$. The readout architecture is designed for low noise and low power. Power dissipation is $\sim 50 \mathrm{~mW}$ in 32 output mode $(80 \mathrm{~K})$ at $100 \mathrm{kHz}$ pixel readout rate and $40 \mathrm{~mW}$ in 4 output mode. The key benefit of this implementation lies in its simplicity. Some results from characterisation by the UKATC of the ME930

Table 4: Leonardo IR detector ME930

\begin{tabular}{|l|l|l|}
\hline Parameter & Value & Comments/conditions \\
\hline Linearity & $\leq 0.8 \%$ & ROIC contribution, 2.1V pixel dynamic range \\
\hline Read noise & $25 \mathrm{e}-\mathrm{rms}$ & Typical for single CDS \\
\hline $\begin{array}{l}\text { Read noise (multiple } \\
\text { NDR) }\end{array}$ & $5 \mathrm{e}-\mathrm{rms}$ & After 50 NDRs \\
\hline Conversion gain & $6 \mu \mathrm{V} / \mathrm{e}-$ & Typical expected \\
\hline Transfer gain & $0.85 \mathrm{~V} / \mathrm{V}$ & Signal chain dominated by pixel source follower gain \\
\hline
\end{tabular}

The pixel circuit includes a clamp function and current limiting during reset in order to minimise the persistence following saturating and/or blooming. Glow (photon emission from active CMOS circuits) is suppressed through the Leonardo standard design approach. Both digital and analogue circuits are designed so that the various metal layers overlap to provide $100 \%$ metal coverage over the active silicon where the glow photons are generated.

Leonardo has extensive experience in the manufacture of hermetically sealed, windowed packages for space applications. A suitable hermetic package for the ME930 could be based on the IASI NG package, which is fully developed. The package includes a flex harness, which could be terminated with a suitable space qualified connector. The ceramic vacuum feedthrough for electrical connections would have to be modified for the ME930 wirebond scheme, and the fixing holes on the metal body would also be adapted for the TWINKLE application. 


\section{FINE GUIDANCE SYSTEM}

In addition to the optimized instrument design to reduce the effect of pointing jitter, a visible Fine Guidance Sensor (FGS) camera is placed at the Instrument Optical Bench downstream from M5 and in reflection of the dichroic and beam-splitter that balances the outputs between FGS and ELVIS. This optical camera will be a standard 1024x1024 CCD with a working field-of-view of \pm 3 arcmin. The camera (or a portion of the array) will be read-out at high-speed and the centroid of the star of interest calculated to better than a tenth of a pixel ( $\sim 0.03$ '). In-flight calibration and commissioning will allow to establish the corresponding working position for the mask slits on the FGS in order for the tip-tilt mirror (TTM) control electronics to keep the source stable within the slit.

Pointing jitter typical of the platform and first derivative of known sources of pointing drifts, allow the TTM to perform well within operating parameters with a payload-in-the-loop arrangement only needed where the source is at risk of exiting the corrected FoV of the FGS, reducing complexity in the payload-platform communication.

The TTM mechanism is based on the heritage design of the Herschel Beam-Steering Mechanism (BSM) [8]. The size of the mirror is almost identical with a similar hole at the aperture plane which will allow the presence of a calibration source if warranted by further simulations. The active mechanism requirements can be relaxed in comparison with the Herschel model given the higher temperature $(>120 \mathrm{~K})$ compared to the $\sim 4 \mathrm{~K}$ version. Finally, the mechanical frame and balancing weight of the TTM will be increased in size to account for the shift in axis of one of the two mechanisms (tilt) given that the angled focal plane requires a simultaneous de-focus to avoid enlarging of the PSF at the masks.

\section{SYSTEM THERMAL DESIGN AND CRYOGENICS}

The overall mechanical design is built on the main requirements of optical rigidity necessary in order to avoid optical distortions, with a view to the implications on the cooling of the system to LEOambient cryogenic conditions on the outside and lower cryogenic temperatures via mechanical cooling on the instrument side. The design is also envelope-constrained from the main platform (SSTL-300) volume and mass requirements, as well as the more detailed potential interface points from payload to platform. The architectural philosophy is to build the structure allowing modular disassembly in view of the aggressive Assembly, Integration and Verification schedules.

\subsection{Telescope Optical Bench and Platform Interface}

The telescope optical bench is the back bone of the telescope. It ties together the entrance baffle (which supports the secondary mirror laterally), the primary mirror, M3 and M4, and the instrument optical bench, including the additional beam splitters and dichroics. The interfaces of directly mounted parts or assemblies are all kinematic.

The TOB is mounted off a bulkhead that interfaces with the spacecraft, kinematically suspended and thermally isolated from the $\mathrm{S} / \mathrm{C}$ body. 


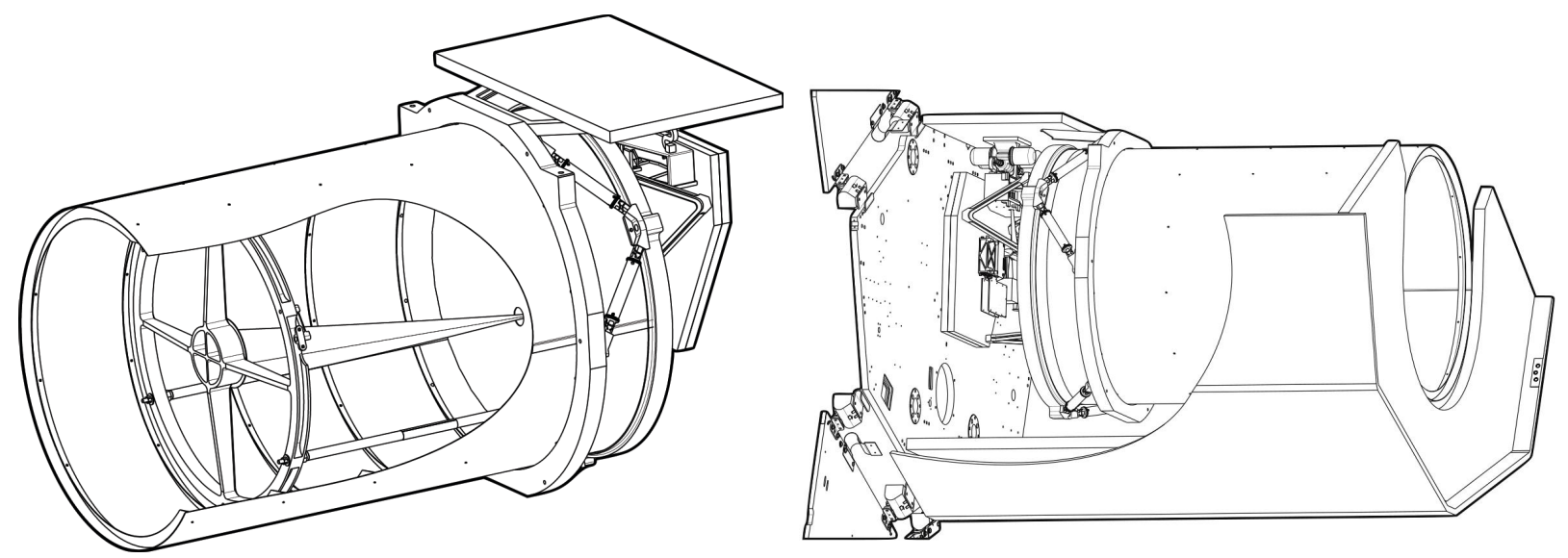

Figure 6-1. Payload assembly with a cut-in view of the telescope baffle and secondary mount. The cooler-dedicated radiator panel is visible at the top, while the telescope baffle (shielded by the platform lateral vanes) will be painted black and act as its own radiator.

The Baffle tube is connected to the T.O.B. at its base with a three-point mounting. It slots into a trough that serves as a stray light baffle. The secondary mirror is mounted to the T.O.B on three Metering Rods. A further three flexural stiffeners are connected tangentially between the M2 mount and the wall of the Baffle Tube.

The Telescope Optical Bench is connected to the Bulkhead via 3 bipods. There are 3 points of connection on the bench and 6 around the circumference of the bulkhead which sits outside of the Baffle Tube. The bipod members will be composed of Torlon (polyamide-imide).

The specific type of Torlon selected here is glass filled and is very stable, no creep has been observed in previous applications. It should be noted that the alignment of the telescope as a whole does not depend on this particular set of bipods. These bipods have been optimised for a combination of stiffness and thermal insulation.
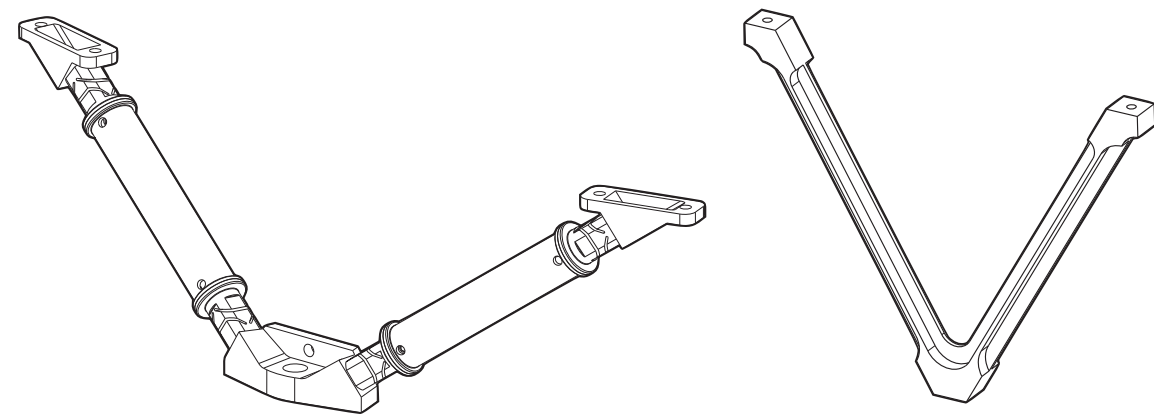

Figure 6-2. Bulkhead bipod (left) and I.O.B. bipod (right).

The IOB is mounted to the TOB via three bipods, providing for a kinematic suspension. There are at present no stringent thermal insulating requirement for this particular interface. The kinematic suspension is optimised for stiffness only. 


\subsection{Mini-coolers}

The coolers adopted to provide the necessary working temperature for the IR detectors (70K) and the reduced operating temperature for the instrument optics and telescope are two Stirling-cycle minicoolers developed at Rutherford Appleton Laboratory Technology department (RAL-Tech) under an ESA Technology Readiness Programme contract. The small scale cooler prototype is shown in Figure 6-3 together with a plot showing the linear scale of heat lift versus the temperature of operation of the cold head.
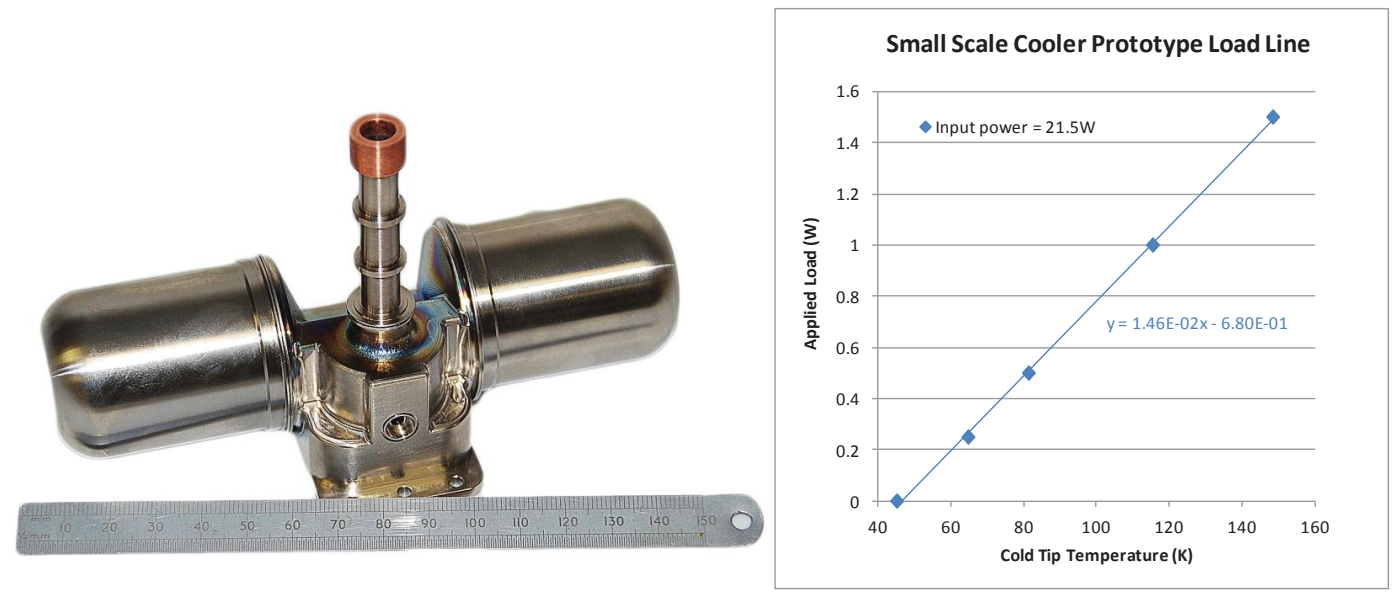

Figure 6-3. RAL-Tech small scale cooler coolers for small missions. Right: Demonstrated heat lift as a function of cold finger temperature under thermal vacuum conditions $-21.5 \mathrm{~W}$ of input power.

The main characteristics of the mini-coolers developed at RAL-Tech which are optimal for the necessary cooling of the IR detectors and inner sanctum are summarized in Table 5.

Table 5. Ral-Tech mini cooler specification summary.

\begin{tabular}{|l|l|}
\hline Mass & $620 \mathrm{~g}$ \\
\hline Size & $145 \mathrm{~mm} \times 70 \mathrm{~mm} \times 95 \mathrm{~mm}$ \\
\hline Input Power & $22 \mathrm{~W}$ \\
\hline Heat Lift & $500 \mathrm{~mW} @ 77 \mathrm{~K}$ \\
\hline Stability (without PID control) & $\pm 5 \mathrm{mK} \mathrm{10mins,} \pm 30 \mathrm{mK} 1 \mathrm{hr}$ \\
\hline mVib (unbalanced displacer) & $3 \mathrm{Npk} @ 90 \mathrm{~Hz}$ \\
\hline mVib (two back-to-back) & $0.3 \mathrm{Npk} @ 90 \mathrm{~Hz}$ \\
\hline Cooler Control Electronics (CCE) & Open loop simple COTS controller \\
\hline
\end{tabular}

The Stirling cycle cooler comprises a pair of linear motor reciprocating compressor units and a single, similarly driven, displacer unit orthogonal to the compressor axis. In the prototype small scale cooler the displacer is unbalanced, although a momentum compensator is commonly employed on larger versions of the cooler. The life and reliability of the cooler stems from the philosophy nonlubricated, non-contacting moving parts, that are enabled by a flexure bearing suspension system operating in the infinite fatigue strength regime. 
The current status of the cooler development is that operation under thermal vacuum environment has been demonstrated and some life confidence data has been obtained ( $>5000 \mathrm{hrs})$.

\subsection{Cooler supporting structure and mounting points}

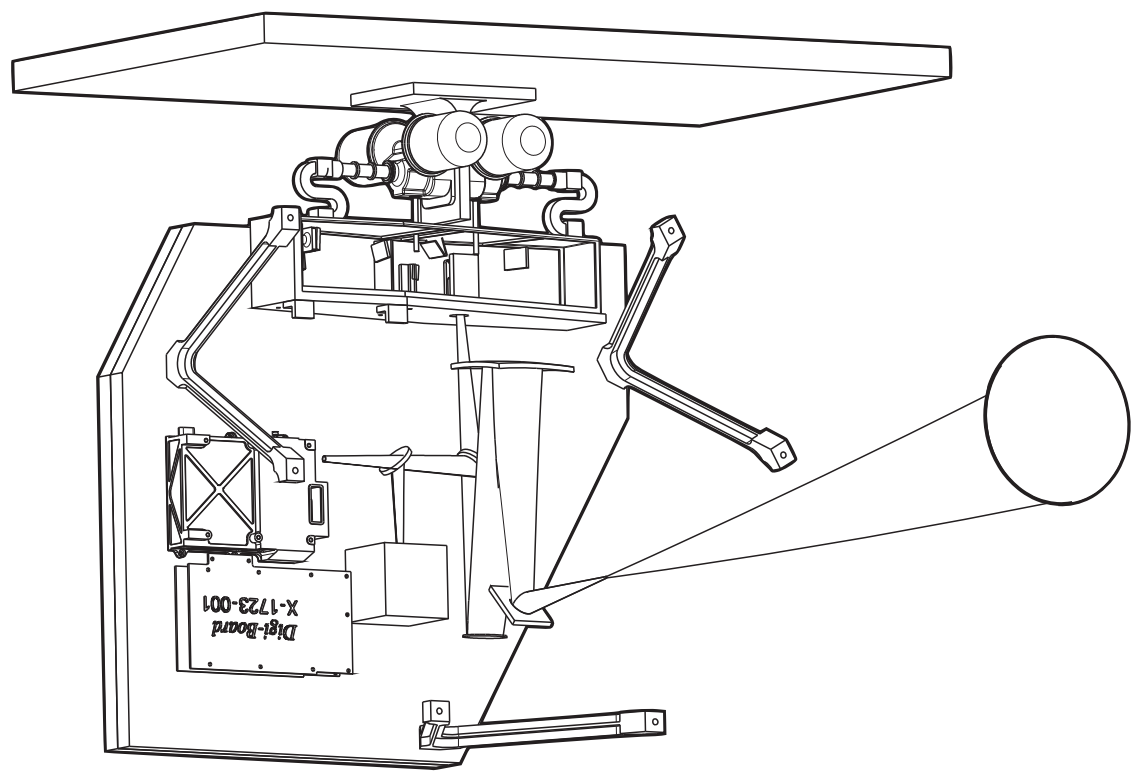

Figure 6-4: Cooler supporting structure

The Coolers are connected on to the radiator by an aluminium mount and are mounted back-to-back to mitigate (and potentially cancel out) the noise generated by mechanical pulse action. The current interface has a thermal contact via aluminium foil thermal straps. One of the cold heads is connected to the IR instrument housing to provide cooling for the overall instrument and through that to the IOB. The other cold head will be connected through an S-slot to the detector mount to provide efficient cooling to a temperature of a approximately $70 \mathrm{~K}$.

The panel radiator with the task of dumping the heat generated by the warm end of the coolers is mounted to the bulkhead. The radiator is in this way mechanically separated from the instrument and is used to provide for a cold sink for the coolers mounted of it. The coolers are connected to the instrument via thermal straps as mentioned before. The area of the radiator is $0.175 \mathrm{~m}^{2}$ (currently implemented as a rectangle $550 \mathrm{~mm} \times 320 \mathrm{~mm}$ ). There is no need for a strict kinematic connection between the radiator and the payload, however one does have to be mindful not to introduce significant loads into the bulkhead when the radiator cools down. A semi-kinematic suspension is considered at present with regard to this particular interface

\section{THE MODIFIED SSTL-300 PLATFORM}

The platform must physically accommodate a payload assembly with an aperture of $52 \mathrm{~cm}$ and length of $85 \mathrm{~cm}$. The instrument includes cryo-coolers and a dedicated radiator to maintain the telescope to its required operating temperature $<200 \mathrm{~K}$. The total mass of the payload is $100 \mathrm{~kg}$ with an average 
power requirement of $100 \mathrm{~W}$, including all margins. The mission will operate in a 600 to $700 \mathrm{~km}$ sun-synchronous dawn-dusk orbit with the boresight of the telescope pointed within a $40^{\circ}$ cone centred around the anti-sun vector.

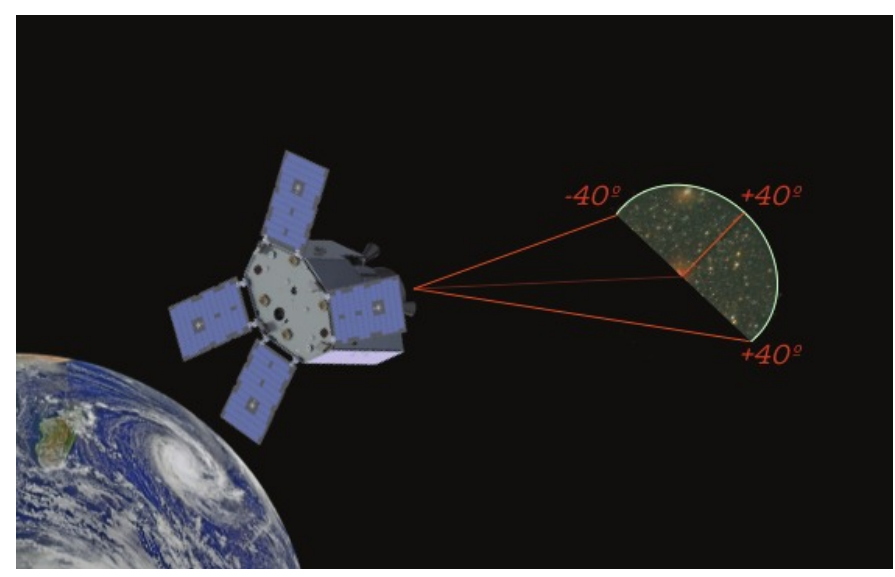

Figure 7-1. Instantaneous Field of Regard of Twinkle.

The "standard" SSTL-300 platform has been modified to include deployable solar panels (deploying mechanism has already been flown), and the communication antennas re-positioned given the nonEO orientation of the spacecraft during operations. Options regarding a potential small sun-shield that shades the radiators on the top-side (Figure 6-1) are being considered.

The core operations and avionics for the platform are from the range used on the majority of SSTL100, SSTL-150 and SSTL-300 missions. These have the advantage of not only being flight proven in similar operating environments to Twinkle, but they have also been operated together routinely across a range of mission types. This means that platform avionics development effort is limited to the payload interface.

Using the SSTL core avionics suite also allows Twinkle to leverage the CONOPS (Concept of Operations) and Fault Detection, Isolation and Recovery (FDIR) approach that has been developed and proven over several previous generations of SSTL spacecraft. Twinkle therefore benefits from the streamlined operations approach, a system designed for robustness and safety, plus high levels of availability all of which have been refined over three decades. A further description of platform considerations is published in [9].

Key features of the CONOPS and FDIR are therefore:

- Robustness and redundancy; simple and robust operational modes that deliver competitive payload availability performance with multiple back-up functionality and equipment on board to assure mission lifetime and guard against unforeseen and random outages and failures.

- On-board autonomy and simple satellite safe-modes, resulting in the elimination of the need for expensive, constantly manned ground segments.

- Extensive use of proven, off-the-shelf components for both platform and payload and ground segment.

- Modularity; investing the development of only a few key new systems that can be arranged in configurations to deliver a wide variety of performance and capacity variations depending on mission requirements.

The current spacecraft system block diagram is shown in Figure 7-2. 


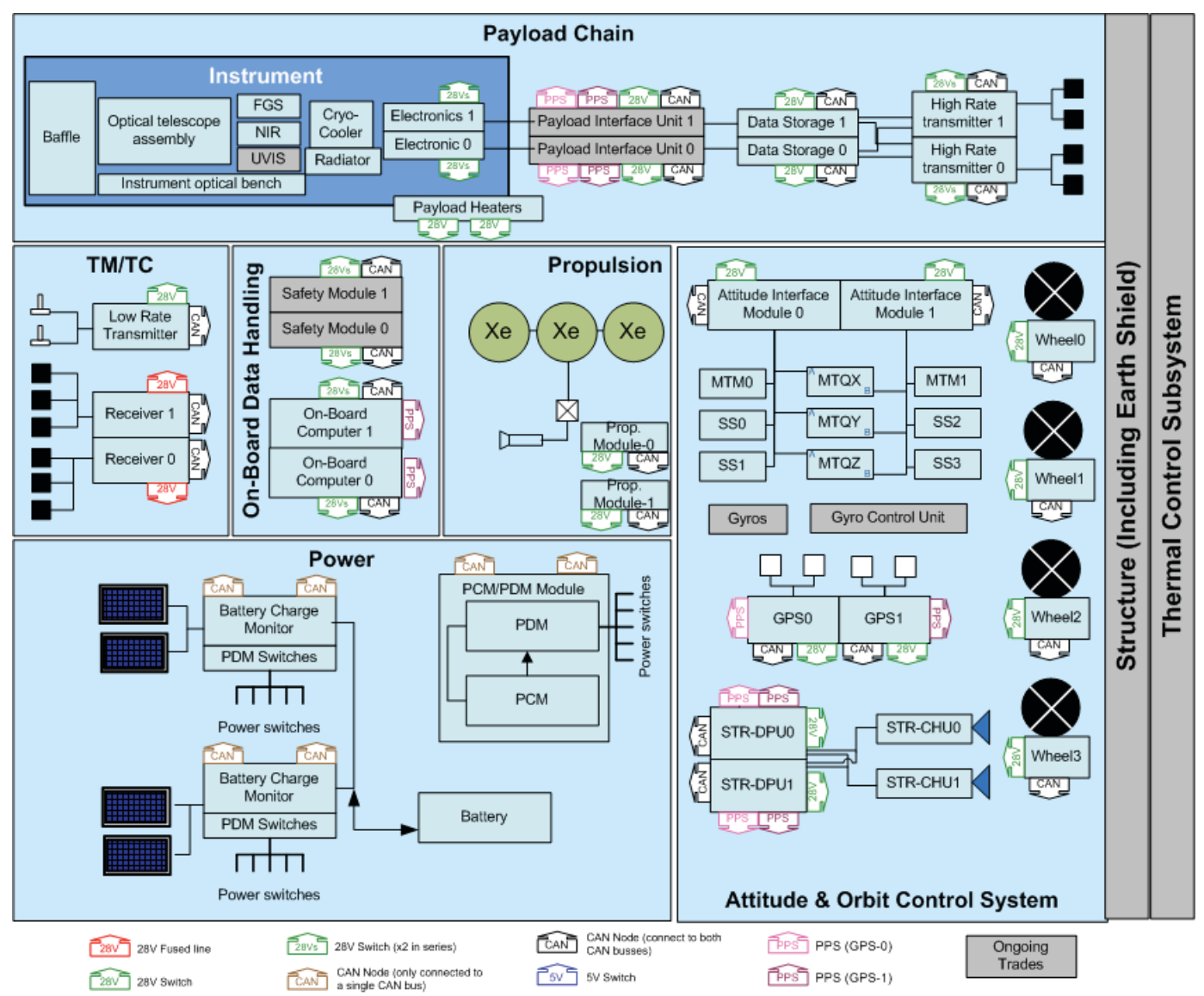

Figure 7-2 Current Twinkle Spacecraft System Functional Block Diagram (trade-offs in progress in grey)

\section{CONCLUSIONS}

In this study we have shown that a commercial Low-Earth Orbit platform can be repurposed for high precision astrophysical observations. Our design benefits from the implementation of existing designs, processes and commercially available components, enabling Twinkle to be built at a low cost and short timescale. The project is currently on track for a launch in 2019 and will be able to deliver the science case it has been designed for. Twinkle will demonstrate that a new approach is possible for the astrophysical sciences, an approach where scientists from countries with or without access to a space program can use facilities to perform astrophysical measurements from space. These facilities can be designed in a relatively short timescale thanks to the high heritage and offthe-shelf components approach, avoiding the long timescales typically associated with astrophysical space missions.

\section{ACKNOWLEDGMENTS}

The authors acknowledge support in part by the ERC under the Advanced Investigator Project 267219, support by Cardiff University and by Surrey Satellite Technology Ltd. 


\section{REFERENCES}

[1] Tessenyi, M., Tinetti G., Tennyson J., Savini G., Pascale E., Jason S., Liddle D., Williams J., Vora A., Saunders C., "TWINKLE: A British space mission to explore faraway worlds", Proc. Of IAC, 2015, IAC-15-B4.2.1

[2] Tinetti G., Encrenaz T., Coustenis A., "Spectroscopy of planetary atmospheres in our Galaxy", Astronomy and Astrophysics Review, v.21, 63 (2013)

[3] Tessenyi, M., Tinetti G., Savini G., and Pascale E. "Molecular Detectability in Exoplanetary Emission Spectra", Icarus, v.226, 2, pp.1654-1672 (2013)

[4] Tinetti, G., Drossart P. (Guest editors), "Topical issue on EChO - the Exoplanet Characterisation Observatory", Experimental Astronomy, v.40, Issue 2-3, 26 articles (2015) - http://link.springer.com/journal/10686/40/2/

[5] Sarkar S. et al. 2016, Proceedings of the SPIE 2016, Submitted

[6] Tyc G., Larson W., Butlin T., Waltham N., Morris N., Tosh I., "High Performance Optical Imaging Payloads for smallsat missions" Proc. Of $22^{\text {nd }}$ Annual AIAA/USU Conference on small satellites SSC08-VI-6, (2008).

[7] Wells M. 2016, Proceedings of the SPIE 2016, Submitted

[8] Pain I., Stobie B., Wright G.S., Paul T.A., Cunningham C.R., "SPIRE beam steering mirror: a cryogenic 2 axis mechanism for the Herschel Space Observatory" Proceedings of the SPIE, Volume 4850, pp. 619-627 (2003).

[9] Jason S., da Silva Curiel A., Tessenyi M., Tinetti G., Savini G., Tennyson J., Pascale E., Williams J., Johnson G., Prasad S., Vora A., Saunders C., Friend J., Sweeting M., "TWINKLE: A new idea for commercial astrophysics missions" Proc. Of 4S Symposium 2016, Submitted. 